\title{
MiR-1179 is downregulated in cervical cancer and its overexpression suppresses cancer cells invasion by targeting CHAF1A/ZEB 1
}

\author{
Fahui Lv', Youwen Zhong'2, Ling Sang'1 and Xiaoling $\mathrm{Wu}^{3 \otimes}$ \\ 1Department of Obstetrics and Gynaecology, The No.2 People's Hospital of Hefei, Hefei, Anhui Province, 230011, China; 2School of Economics \\ and Finance, Xi'an Jiaotong University, Xi'an, Shaanxi Province,710061, China; ${ }^{3}$ Department of Obstetrics and Gynecology, The Second Affiliated \\ Hospital of Xi'an Jiaotong University, Xi'an, Shaanxi Province, 710004, China
}

The anticancer effect of miR-1179 has been extensively studied in many tumors. The mechanism of miR-1179 action in cervical cancer, however, remains largely unknown. In the present study, miR-1179 was downregulated in both cervical cancer cell lines and cancer tissues. In addition, miR-1179 mimic suppressed cancer cells invasion and epithelial-mesenchymal transition (EMT) in cervical cancer $\mathrm{SiHa}$ and Caski cells. We found that chromatin assembly factor 1 subunit A (CHAF1A) might be a direct target of miR-1179 and could be regulated by miR-1179. Furthermore, CHAF1A shRNA suppressed the cervical cancer cells invasion and the expression of EMT-promoted proteins. Reversely, CHAF1A overexpression not only promoted cervical cancer cells invasion but also upregulated the level of Zinc finger E-box binding protein 1 (ZEB1), an EMT-related protein. The induction of ZEB1 could be counteracted by miR-1179 overexpression. It was observed that in cervical cancer patients' tissues, miR-1179 was downregulated while the pathway of CHAF1A/ZEB1 was upregulated. In summary, our research indicated that the miR-1179 might regulate CHAF1A/ZEB1 axis and inhibit the invasion of cervical cancer cells.

Keywords: cervical cancer, miR-1179, epithelial-mesenchymal transition, CHAF1A/ZEB1

Received: 23 September, 2020; revised: 26 October, 2020; accepted: 30 October, 2020; available on-line: 19 March, 2021

घe-mail: xlwu666@163.com

Abbreviations: CHAF1A, chromatin assembly factor 1 subunit $A_{\text {; }}$ EMT, epithelial-mesenchymal transition; HPV, human papillomavirus; ZEB1, Zinc finger E-box binding protein 1

\section{INTRODUCTION}

Cervical cancer is one of the most common gynecological malignancies caused by human papillomavirus (HPV) infection. Clinical trials has validated that HPV vaccines reduce the risk of cervical cancer with HPV type 16 and 18 by $70 \%$ and by and $86-95 \%$ for other HPV-associated cancers (Markham et al., 2020). However, new cases of cervical cancer still account for $10 \%$ of all gynecological cancers and approximately $5 \%$ of all tumors (Hovland et al., 2010), second only to breast cancer (Ferlay et al., 2010; De Martel et al., 2012), with more than $85 \%$ of cervical cancers prevalent in developing countries and more than $80 \%$ of all cases (Arbyn et al., 2011). Epithelial-mesenchymal transition (EMT) and cancer cells invasion might be the main reasons for cervical cancer malignancy. Therefore, in addition to the prevention of cervical cancer with HPV vaccines, biological biomarkers and therapeutic targets have gained increasing interest as there is still the demand for the treatment of cervical cancer.

MicroRNAs (miRNAs) are small non-coding RNAs that regulate gene expression by inhibiting post-transcriptional translation of mRNA. The relationship between miRNA and tumors has been extensively studied, and many studies found that various miRNAs play an important regulatory role in the occurrence and development of cervical cancer (Wilting et al., 2010; Wang et al., 2011; Liu et al., 2012; Xie et al., 2012; Zhu et al., 2013). The anticancer effect of miR-1179 has been extensively studied in many tumors (Lin et al., 2018; Song et al., 2018; Gao et al., 2019; Li \& Qin 2019), but its pro-oncogenic effect was also reported (Jiang et al., 2015; Zhihong et al., 2019). However, the effect as well as the underlying mechanism of miR-1179 action in cervical cancer remain unknown. Therefore, we aimed to investigate the expression level and the effect of miR-1179 in cervical cancer.

Chromatin assembly factor 1 subunit A (CHAF1A) is a pro-oncogene that plays a role in a variety of tumors, such as colon cancer (Wu et al., 2014), glioblastoma (Peng et al., 2016), ovarian cancer (Xia et al., 2017) and gastric cancer (Zheng et al., 2018). In addition, studies showed that the expression of CHAF1A was increased in cervical cancer and was positively correlated with tumor malignancy (Luo et al., 2017). However, the specific mechanism of its action in cervical cancer needs to be further studied. Interestingly, in the present study, we found that miR-1179 could bind to the 3'UTR of CHAF1A. Furthermore, CHAF1A overexpression promoted EMT and invasion. Thus, we speculated that CHAF1A might be a regulatory target of miR-1179 in cervical cancer. Zinc finger E-box binding protein 1 (ZEB1) was reported as EMT-activator in cancer biology (Zhang et al., 2015). In previous studies, CHAF1A was demonstrated to interact with TCF4, which promotes gastric carcinogenesis (Zheng et al., 2018), while $\beta$-catenin/ TCF4 complex can induce the EMT-activator ZEB1 to regulate tumor invasion (Sánchez-Tilló et al., 2011). Moreover, TCF4 can regulate ZEB1/ZEB2 expression in drug resistance and stemness of colorectal cancer (Sun et al., 2020). These studies indicated that a regulatory mechanism might exist for CHAF1A and ZEB1. In summary, we aimed to clarify the action of miR-1179/CHAF1A/ZEB1 axis in cervical cancer and this study might provide a new target for cervical cancer therapy. 


\section{MATERIALS AND METHODS}

\section{Cell lines and culture}

The cervical cancer cell lines $\mathrm{SiHa}$ and Caski were purchased from the American type culture collection (ATCC; Rockville, MD, USA). SiHa cells were cultured in Eagle's Minimum Essential Medium (EMEM, Gibco, Grand Island, NY, USA) and Caski cells were cultured in RPMI-1640 Medium (Gibco), respectively, supplemented with $10 \% \mathrm{FBS}$ (Gibco) in an incubator at $37^{\circ} \mathrm{C}$ with $5 \% \mathrm{CO}_{2}$.

\section{Cell transfection}

MiR-1179 mimic (miR-1179 overexpression), negative control (NC mimic), pGL3-CHAF1A-wild type (WT), pGL3-CHAF1A-mutant (MUT), negative control (shNC), shCHAF1A (CHAF1A knockdown) and pcDNA3.1-CHAF1A (CHAF1A overexpression) were obtained from GenePharma Co., Ltd. (Shanghai, China). The vectors were separately transfected into cells using Lipofectamine 3000 (Invitrogen, Carlsbad, USA) strictly according to the manufacturer's instruction. After transfection, cells were cultured for $48 \mathrm{~h}$ and quantitative polymerase chain reaction (qPCR) was conducted to assess the transfection efficiency, and cells with satisfactory transfection efficiency were used for the subsequent experiments.

\section{qPCR}

Total RNA of SiHa and Caski cells and cervical cancer tissues was extracted with TRIzol reagent according to the manufacturer's protocols (Invitrogen). Briefly, $2 \mu \mathrm{g}$ of RNA was reversely transcribed into cDNA with SuperScriptII Reverse Transcriptase kit (Invitrogen). Then the expression levels of miR-1179 and CHAF1A were quantified using SYBR Green supermix (Bio-Rad Corp.,USA) in the PCR 7300 System (Applied Biosystems, Foster City, USA). Primers for miR-1179, snRNA U6, $\beta$-actin, ZEB1 and CHAF1A were synthesized by Sangon Biotech (Shanghai, China). Primers were as follows: ZEB1 (Peña et al., 2006): forward primer: 5'-GCCAATAAGCAAACGATTCTG-3' and reverse primer: 5'-T'T'TGGCTGGATCACT'TCAAG-3'; miR-1179: forward primer: 5'-GGCTGGAAAGGAAGAAG-3'; reverse primer: 5'-GT'TAACCCGGCGGTGA-3'; CHAF1A (Liu et al., 2017): forward primer: 5'-AGGGAAGGTGCCTATGGTG-3' and reverse primer: 5'-CAGGGACGAATGGCTGAGTA-3'; U6: forward primer: 5'-CTCGCTTCGGCA GCACA-3' and reverse primer: 5'-AACGCT'TCACGAAT'TTGCGT-3'; $\beta$-actin (Li et al., 2016): forward primer: 5'-GAGCTACGAGCTGCCTGACG-3' and reverse primer: 5'- GTAGT'TTCGTGGATGCCACAG-3'. The qPCR data were analyzed by comparative Ct method and $2^{-\Delta \Delta C T}$ method. For control, miR-1179 expression was normalized to U6 while CHAF1A and ZEB1 expression was normalized to $\beta$-actin.

\section{Transwell assay}

$\mathrm{SiHa}$ and Caski cells were inoculated into the upper Transwell chambers filled with serum-free EMEM and RPMI-1640, respectively, at a density of $5 \times 10^{4} / 200 \mu \mathrm{L}$. At the same time, $500 \mu \mathrm{L}$ EMEM and RPMI-1640, respectively, containing 10\% FBS were placed in the lower Transwell chambers (Chen et al., 2019) . After 24 h of culture, the cells in lower chambers were fixed with $95 \%$ ethanol and stained with crystal violet. Finally, the invasive cells were counted under a light microscope. The experiments were repeated 3 times.

\section{Western blot assay}

48 Hours after transfection, the protein expression of EMT markers (E-cadherin, N-cadherin), CHAF1A, ZEB1 and $\beta$-actin (Abcam, Cambridge, UK) was detected via Western blot. The expression level of target proteins was normalized to the expression of $\beta$-actin. The Western blot protocol was performed according to the literature (Lee \& Hwang 2017). Target blots were visualized using the Chemiluminescent ECL Reagent Kit (Millipore, Bedford, MA, USA).

\section{Luciferase reporter assay}

The CHAF1A 3'UTR wild-type (WT) sequence or mutant binding sites (MUT) complementary to miR1179 were amplified by PCR and cloned to pGL3-Basic vector. For luciferase reporter assays, pRL-TK and WT or MUT versions reporter plasmid vectors or miR-1179 mimics were co-transfected into Caski cells using Lipofectamine 3000 reagent. Cells were cultured for $48 \mathrm{~h}$, and then the relative luciferase intensity was detected by the dual-luciferase reporter assay system (Promega, WI, USA) using a standard protocol. The relative luciferase intensity was normalized to Renilla luciferase intensity.

\section{Immunohistochemical staining (IHC)}

The study protocol was approved by the Ethics Committee of The No.2 People's Hospital of Hefei and performed in accordance with the revised version of the Declaration of Helsinki principles. All cervical cancer patients agreed to the use of their tumor tissues for IHC, and written consent was obtained from all the patients. 3- $\mu \mathrm{m}$ thick tissue sections from cervical cancer patients (including cervical cancer and normal cervical tissue) were prepared. After fixation with formalin and embedding in paraffin, tissue sections were submitted to standard immunohistochemistry processing with antiCHAF1A and anti-ZEB1 antibodies (Abcam). The samples were observed and $(\times 400)$ photographed under an optical microscope (Olympus).

\section{Statistical analysis}

Statistical analysis was performed using the statistical software (SPSS; version 17.0; Inc., Chicago, IL). The data were presented as means \pm SD. Statistical differences were analyzed using paired t-test or one-way ANOVA followed by Tukey's posthoc test. $P$ values $<0.05$ were considered statistically significant.

\section{RESULTS}

\section{Overexpression of miR-1179 inhibited EMT-upregulated proteins and invasion of cervical cancer cells}

In both Caski and SiHa cells, miR-1179 mimic upregulated the expression of miR-1179 compared to NC mimic (Fig. 1A). Overexpression of miR-1179 via miR1179 mimic significantly reduced the number of cells that passed through the Transwell basement membrane (Fig. 1B). Additionally, the N-cadherin protein expression was reduced, whereas the E-cadherin protein expression was increased after miR-1179 mimic transfec- 
$A$
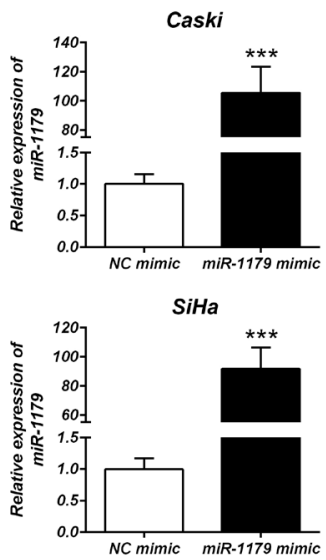

C

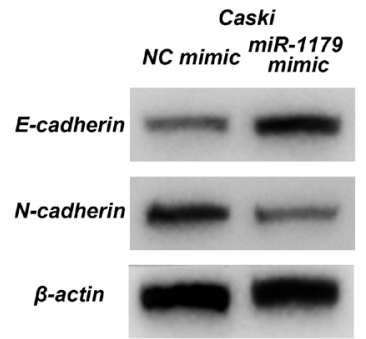

$B$
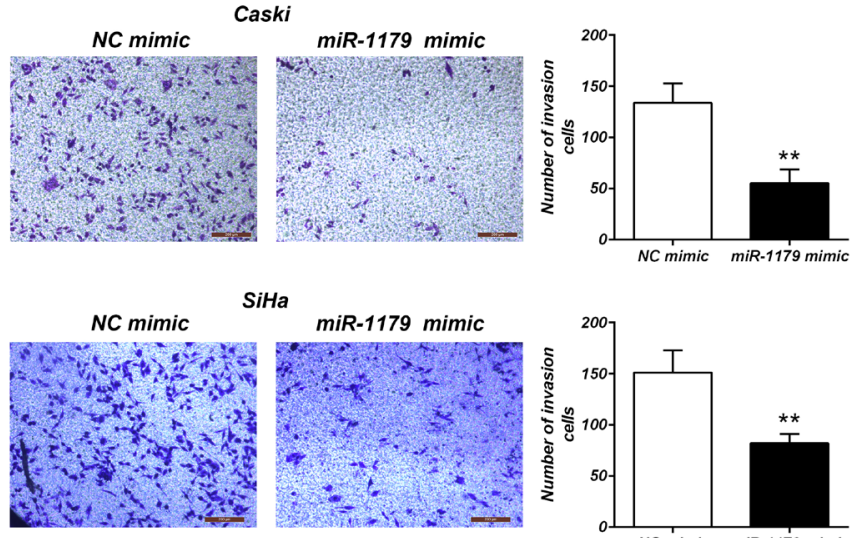

$\mathrm{SiHa}$
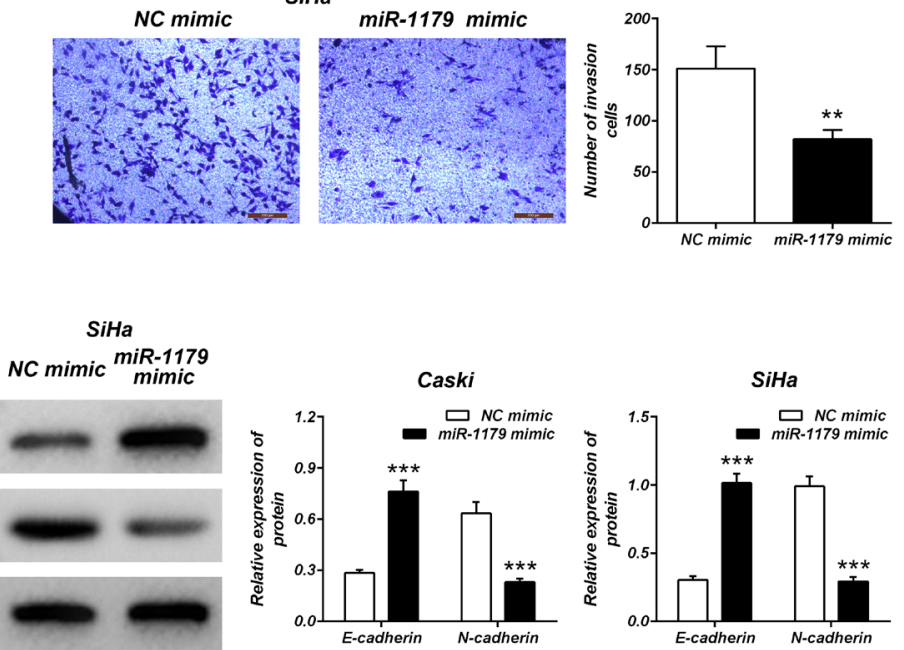

Figure 1. Overexpression of miR-1179 inhibited the invasion of cervical cancer cells.

Caski and SiHa cells were transfected with NC mimic or miR-1179 mimic. (A) qPCR was used to detect the expression of miR-1179 in Cas$\mathrm{ki}$ and $\mathrm{SiHa}$ cells. (B) Transwell assay was performed to assess the invasion of Caski and SiHa cells. (C) Western blot assay was performed to detect the protein expression of EMT-related proteins. The experiments were repeated three times. ${ }^{* *} p<0.01,{ }^{* * *} p<0.001$ compared to the NC mimic group.

tion (Fig. 1C). These results indicated that overexpression of miR-1179 inhibited the invasion ability and EMT-upregulated proteins of Caski and SiHa cells.

\section{Targeted binding of miR-1179 to CHAF1A}

During the investigation of the potential targets of miR-1179 using TargetScan, miR-1179 was predicted to bind to the CHAF1A 3'UTR (Fig. 2A). MiR-1179 targeting CHAF1A was then verified by double-luciferase reporting assay (Fig. 2B). The expression of miR-1179 was vastly inhibited by miR-1179 inhibitor (Fig. 2C). In addition, the mRNA level of CHAF1A was decreased upon the treatment with miR-1179 mimic but was increased by miR-1179 inhibitor (Fig. 2D). The same trends were found at the protein level (Fig. 2E). These results suggested that CHAF1A was a target gene of miR-1179 and it could be regulated by miR-1179.

\section{CHAF1A knockdown inhibited ZEB1 expression}

To reveal the regulatory mechanism of CHAF1A and ZEB1, we performed CHAF1A knockdown using shCHAF1A to detect the level of ZEB1 in Caski and $\mathrm{SiHa}$ cells. In both Caski and $\mathrm{SiHa}$ cells, CHAF1A knockdown inhibited ZEB1 expression both at the mRNA and protein level (Fig. 3A-B).

CHAF1A overexpression reversed the effect of miR-1179 mimic on cell invasion

To further explore the mechanism of miR-1179/ CHAF1A axis in cervical cancer, both Caski and $\mathrm{SiHa}$ cells were transfected with miR-1179 mimic, CHAF1A
-pcDNA-3.1 vector, or miR-1179 mimic+CHAF1A -pcDNA-3.1 vector. Consequently, miR-1179 mimic decreased the levels of CHAF1A, ZEB1 and Ncadherin, but upregulated E-cadherin expression. On the contrary, CHAF1A overexpression significantly upregulated CHAF1A, ZEB1 and N-cadherin, but downregulated E-cadherin (Fig. 4A). As expected, the CHAF1A overexpressing cells treated with miR-1179 mimic showed no such effects (Fig. 4A), suggesting that miR-1179 might inhibit EMT-upregulated proteins by suppressing CHAF1A and ZEB1 expression. Additionally, the inhibitory effect of miR-1179 on the invasion of both Caski and $\mathrm{SiHa}$ cells was reversed by CHAF1A overexpression (Fig. 4B), which further confirmed that miR-1179 might inhibit cell invasion by downregulating the expression of CHAF1A and ZEB1.

\section{MiR-1179 was downregulated in tumor tissues and negatively correlated with the expression of CHAF1A and ZEB1}

As shown in Fig. 5 A\&C, the relative mRNA level of miR-1179 was decreased in cervical cancer tissues compared to the normal tissues of patients. Conversely, the relative expression of CHAF1A and ZEB1 was elevated in cervical cancer tissues compared to the normal tissues of patients. In addition, the Pearson analysis revealed a negative correlation between the expression of CHAF1A1 and miR-1179 (Fig. 5B, $r=0.7108, P<0.001$ ), and a negative correlation between the expression of ZEB1 and miR-1179 (Fig. 5B, r=0.5196, $P<0.001$ ) in cervical cancer tissues. On the other hand, a positive correlation between the expression of ZEB1 and 
$\boldsymbol{A}$

PGL3-CHAF1A-WT

hsa-miR-1179

pGL3-CHAF1A-MUT
5'...GGGGUGACGUAUGUAGAAUGCUU...3'

3' gguvgguUacuuUcuuacgaA 5,

5'...GGGGUGACGUAUGUACUUACGAU...3'
B

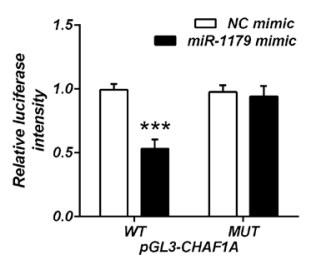

C

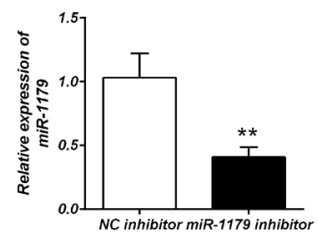

D

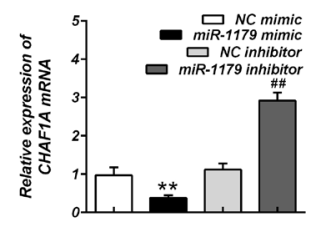

E
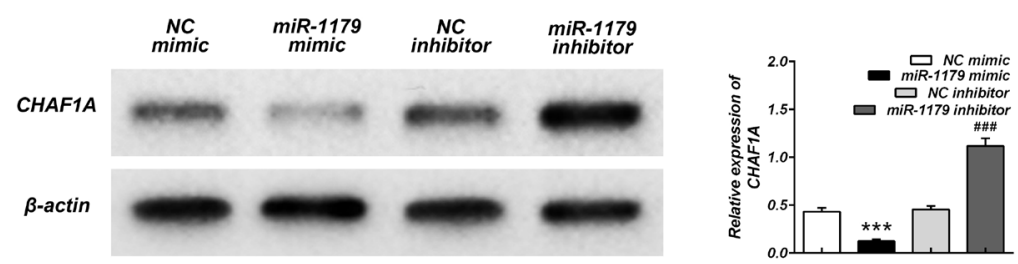

Figure 2. Targeted binding of miR-1179 to CHAF1A.

(A) Target prediction was conducted using the TargetScan website. (B) Caski cells were divided into NC mimic + pGL3-CHAF1A-WT, miR1179 mimic + pGL3-CHAF1A-WT-NC mimic + pGL3-CHAF1A-MUT and miR-1179 mimic + pGL3-CHAF1A-MUT groups. Luciferase reporter assay was performed to confirm the target relationship between miR-1179 and CHAF1A. (C-D) qPCR was used to detect the expression of miR-1179 and CHAF1A in Caski cells. (E) Western blot assay was performed to detect the expression of CHAF1A protein in Caski cells. The experiments were repeated three times. ${ }^{* *} p<0.01,{ }^{* * * *} p<0.001$ compared to the NC mimic group; ${ }^{* \#} p<0.01$, ${ }^{* \#} p<0.001$, compared to the NC inhibitor group.

CHAF1A1 (Fig. 5B, r=0.7378, $P<0.001$ ) was observed in cervical cancer tissues. All these results indicated that CHAF1A might be a target of miR-1179 and the expression of ZEB1 and CHAF1A1 in cervical cancer could be regulated by the miR-1179 level.

\section{DISCUSSION}

The acquisition of EMT features has been demonstrated to be an important contributor to the metastasis of cancer cells ( $\mathrm{Li}$ et al., 2016). In cervical cancer, EMT was identified to play a major role in promoting invasion (Xiao et al., 2018), which is also an essential prerequisite for cancer cell invasion and migration ( $\mathrm{Li}$ et al., 2016). In this study, we explored the influence of miR-1179 in cervical cancer and the regulatory mechanism behind the invasion of cervical cancer cells.

MiRNAs, small non-coding RNAs, have been extensively studied in terms of its association with cancer biology (Wilting et al., 2010; Wang et al., 2011; Liu et al., 2012; Xie et al., 2012; Zhu et al., 2013). In the present study, we found that miR-1179 was downregulated in cervical cancer cells as well as cervical cancer patient tis-
$\boldsymbol{A}$

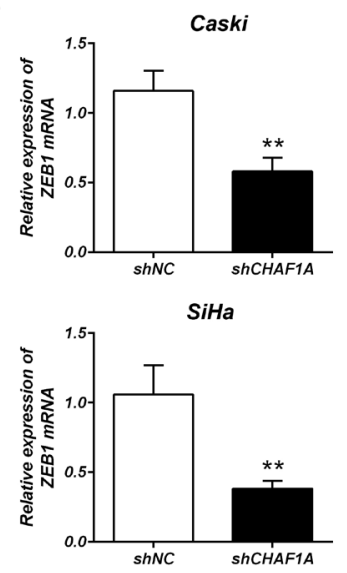

B

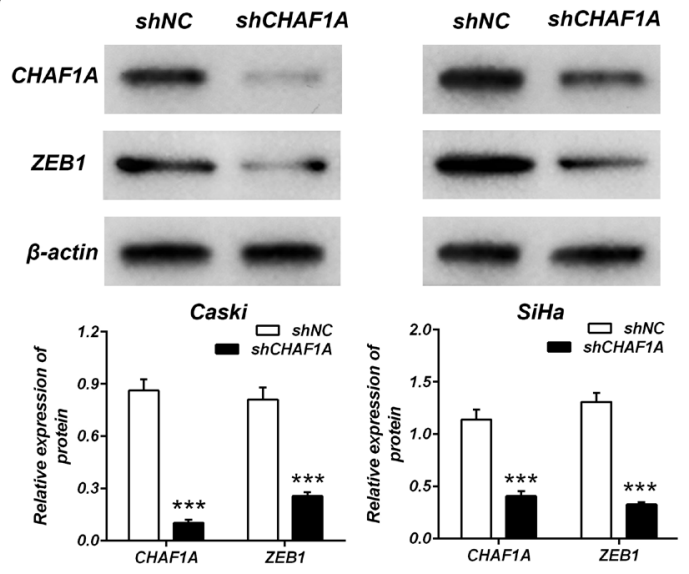

Figure 3. CHAF1A knockdown inhibited ZEB1 expression.

Caski and SiHa cells were transfected with shRNA NC (shNC) or shRNA CHAF1A (shCHAF1A). (A) qPCR was used to detect the expression of ZEB1 in Caski and SiHa cells. (B) Western blot assay was performed to detect the protein expression of ZEB1 and CHAF1A. The experiments were repeated three times. ${ }^{* *} p<0.01,{ }^{* * *} p<0.001$ compared to the NC mimic group. 
$\boldsymbol{A}$
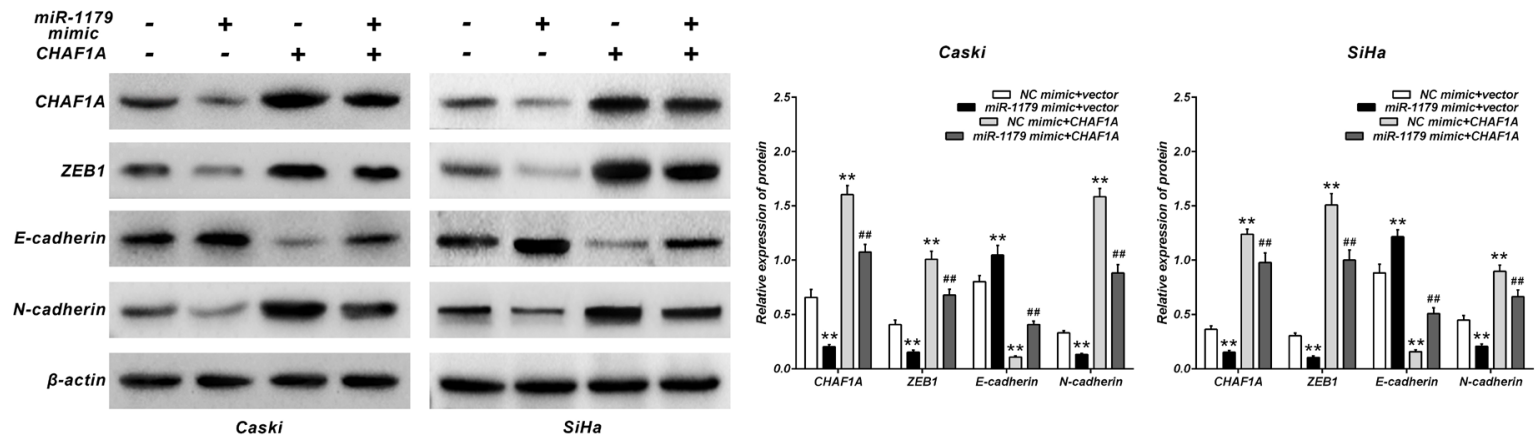

B
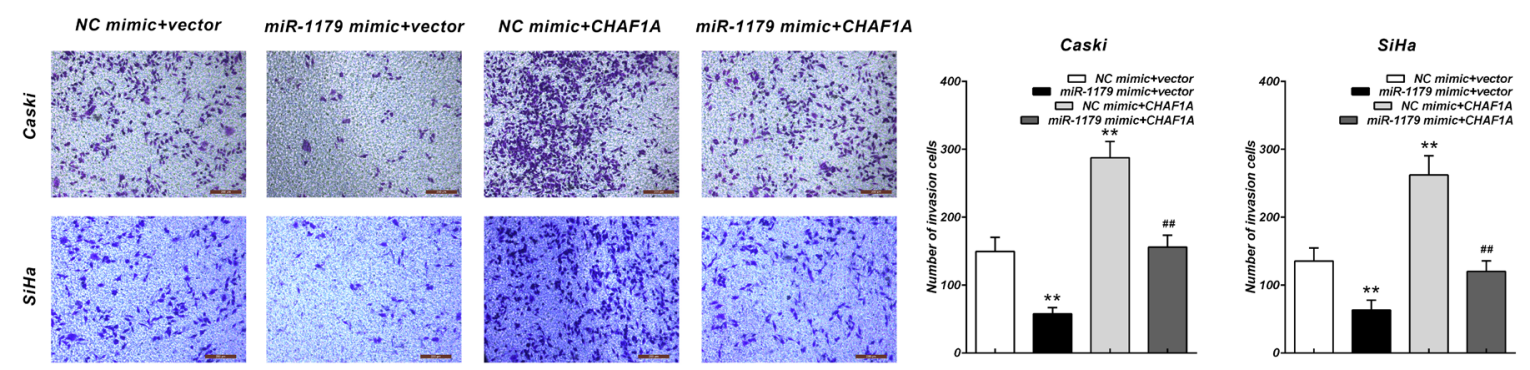

Figure 4. CHAF1A overexpression reversed the effect of miR-1179 mimic on the cells invasion.

Caski and SiHa cells were divided into NC mimic, miR-1179 mimic, NC inhibitor, miR-1179 inhibitor groups. (A) Western blot assay was performed to detect the protein expression. (B) Transwell assay was performed to assess the invasion of Caski and SiHa cells. The experiments were repeated three times. ${ }^{* *} p<0.01$ compared to the NC mimic + vector group; ${ }^{* \#} p<0.01$ compared to the NC mimic + CHAF1A group.

A
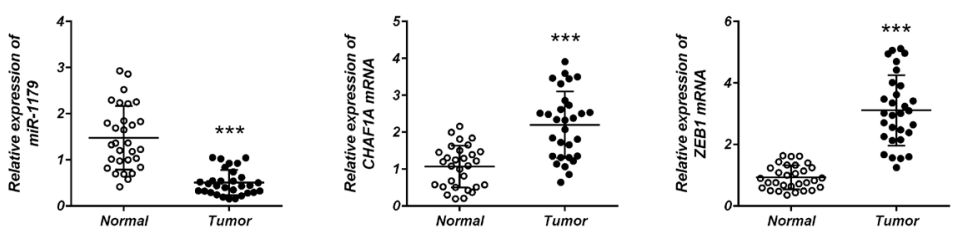

$B$

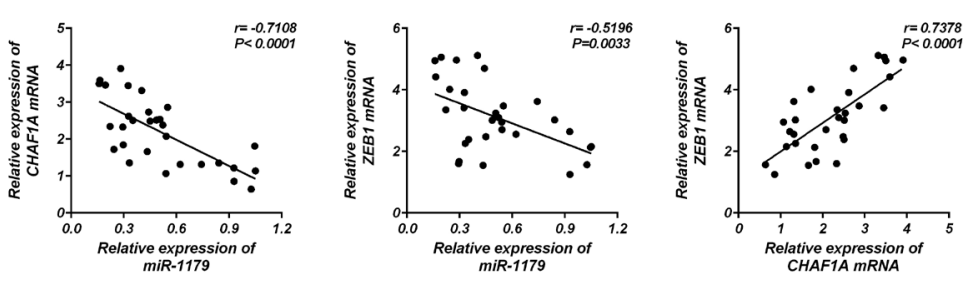

C
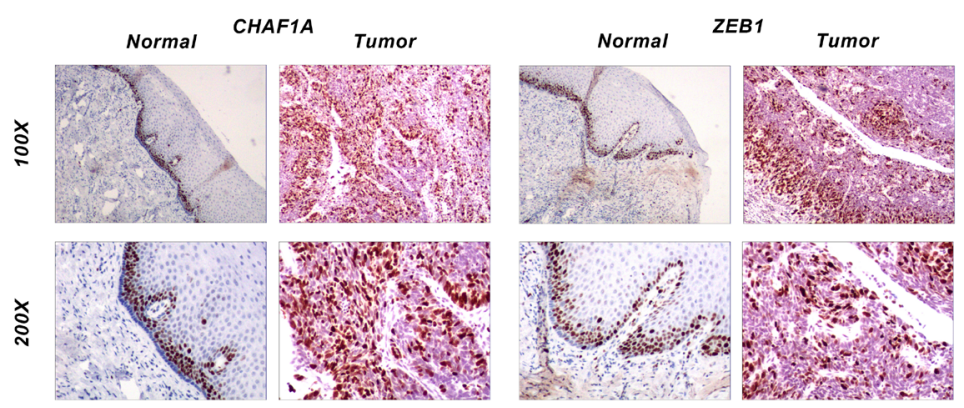

Figure 5. MiR-1179 was downregulated in tumor tissues and negatively correlated with the expression of CHAF1A and ZEB1.

(A) The expression levels of miR-1179, CHAF1A and ZEB1 were detected by GPCR in cervical cancer and normal cervical tissue. ${ }_{* * *} p<0.001$ compared to the normal tissues. (B) Correlation of miR-1179 and CHAF1A was assessed using Pearson analysis. (C) CHAF1A and ZEB1 distribution detected using IHC. The experiments were repeated three times. 
sues. However, contradictory results regarding the effects in cancers were previously obtained for miR-1179. Some researchers indicated that miR-1179 promoted cell invasion in esophageal squamous cell carcinoma (Jiang et al., 2015). MiR-1179 has been also identified as a suppressor in several cancers such as human non-small cell lung cancer (Heller et al., 2018), gastric cancer (Song et al., 2018), papillary thyroid carcinoma (Ye et al., 2020) and cervical cancer (Qu et al., 2020). Interestingly, consistently with the effect of miR-1179 in other cancers, our study also supports the inhibitory role of miR-1179 in cervical cancer, as miR-1179 suppressed the cervical cancer cells invasion. Thus, this result further verified the anti-cancer role of miR-1179 in cervical cancer and suggested that the loss of miR-1179 might induce the malignancy of cervical cancer including cells invasion.

In addition, CHAF1A was predicted as a putative target gene of miR-1179 using TargetScan website, and miR-1179 mimic significantly reduced the mRNA and protein level of CHAF1A. CHAF1A is the main functional subunit of CHAF1 protein (Liu et al., 2017), which is involved in deoxyribonucleic acid (DNA) replication, gene expression regulation and DNA mismatch repair (Cai et al., 2018). It was found that CHAF1A plays an essential role in the occurrence and development of various malignant tumors, and is related to a poor prognosis of cancers. Most importantly, CHAF1A overexpression promotes the migration and invasion of cancer cells (Liu et al., 2017; Zheng et al., 2018). Therefore, CHAF1A, acting as an oncogene, may be a target for the diagnosis and therapy in malignant cancers. CHAF1A overexpression was proved to be positively correlated with the malignancy of cervical squamous cell carcinoma (Luo et al., 2017). We also found that CHAF1A could directly bind miR-1179 in cervical cancer cells.CHAF1A was also significantly upregulated in cervical cancer cells and patient tissues. Consistently with the previous studies, a negative correlation was found between CHAF1A expression and miR-1179 expression. Expectedly, CHAF1A overexpression effectively reversed the inhibitory effect of miR1179 on the invasion of cervical cancer cells. All these results indicated that the inhibitory effect of miR-1179 on cervical cancer invasion was achieved by inhibiting the expression of CHAF1A. ZEB1 was found to play a key role in tumorigenesis and tumor progression. In addition, previous studies demonstrated that ZEB1 contributed to the promotion of EMT (Yu et al., 2018). In this study, similarly to CHAF1A, ZEB1 was overexpressed in cervical cancer cells and tumor tissues. Along with this, a negative correlation was found between ZEB1 expression and miR-1179 expression, while we revealed a positive correlation between the expression of ZEB1 and CHAF1A. However, the mechanism of ZEB1 regulation by CHAF1A is uncertain and needs to be further investigated.

In summary, the current research revealed that miR1179 was downregulated in cervical cancer and its overexpression suppressed cancer cells invasion, while CHAF1A overexpression reversed this inhibitory effect. Moreover, CHAF1A was identified as a direct target of miR-1179 and miR-1179 might regulate cervical cancer through CHAF1A/ZEB1 axis. The present study revealed for the first time the critical role of the miR$1179 / \mathrm{CHAF} 1 \mathrm{~A} / \mathrm{ZEB} 1$ axis in cervical cancer and might provide new targets and theoretical basis for cervical cancer therapy.

\section{Acknowledgements}

Not applicable.

\section{Funding}

Not applicable.

\section{Competing interests}

The authors state that there are no conflicts of interest to disclose.

\section{Ethics approval}

Ethical approval was obtained from the Ethics Committee of The No.2 People's Hospital of Hefei.

\section{Statement of Informed Consent}

Written informed consent was obtained from a legally authorized representative(s) for anonymized patient information to be published in this article.

\section{Availability of data and materials}

All data generated or analyzed during this study were included in this published article.

\section{Authors' contributions}

Fahui Lv designed the study, supervised the data collection, YouWen Zhong analyzed the data, interpreted the data, Ling Sang and XiaoLing Wu prepared the manuscript for publication and reviewed the draft of the manuscript. All authors have read and approved the manuscript.

\section{REFERENCES}

Arbyn M, Castellsagué X, de Sanjosé S, Bruni L, Saraiya M, Bray F, Ferlay J (2011) Worldwide burden of cervical cancer in 2008. Ann Oncol 22: 2675-2686. https://doi.org/10.1093/annonc/mdr015

Cai Y, Dong ZY, Wang JY (2018) MiR-520b inhibited metastasis and proliferation of non-small cell lung cancer by targeting CHAF1A. Eur Rev Med Pharmacol Sci 22: 7742-7749. https://doi.org/10.26355/ eurrev_201811_16396

Chen Q, Chen Z, Cao S, Guo B, Chen Y, Feng Z, Wang J, Guo G, Chen X, Huang X (2019) Role of CircRNAs_100395 in proliferation and metastases of liver cancer. Med Sci Monit 25: 6181-6192. https://doi.org/10.12659/msm.915963

de Martel C, Ferlay J, Franceschi S, Vignat J, Bray F, Forman D, Plummer M (2012) Global burden of cancers attributable to infections in 2008: a review and synthetic analysis. Lancet Oncol 13: 607-615. https://doi.org/10.1016/S1470-2045(12)70137-7

Ferlay J, Shin HR, Bray F, Forman D, Mathers C, Parkin DM (2010) Estimates of worldwide burden of cancer in 2008: GLOBOCAN 2008. Int J Cancer 127: 2893-2917. https://doi.org/10.1002/ijc.25516

Gao HB, Gao FZ, Chen XF (2019) MiRNA-1179 suppresses the metastasis of hepatocellular carcinoma by interacting with ZEB2. Eur Rev Med Pharmacol Sci 23: 5149-5157. https://doi.org/10.26355/eurrev_201906_18179

Heller G, Altenberger C, Steiner I, Topakian T, Ziegler B, Tomasich E, Lang G, End-Pfützenreuter A, Zehetmayer S, Döme B, Arns BM, Klepetko W, Zielinski CC, Zöchbauer-Müller S (2018) DNA methylation of microRNA-coding genes in non-small-cell lung cancer patients. J Pathol 245: 387-398. https://doi.org/10.1002/path.5079

Hovland S, Muller S, Skomedal H, Mints M, Bergström J, Wallin KL, Karlsen F, Johansson B, Andersson S (2010) E6/E7 mRNA expression analysis: a test for the objective assessment of cervical adenocarcinoma in clinical prognostic procedure. Int J Oncol 36: 15331539. https://doi.org/10.3892/ijo_00000640

Jiang L, Wang Y, Rong Y, Xu L, Chu Y, Zhang Y, Yao Y (2015) miR1179 promotes cell invasion through SLIT2/ROBO1 axis in esophageal squamous cell carcinoma. Int J Clin Exp Pathol 8: 319-327. PMID: 25755718

Lee JY, Hwang JY (2017) Analysis of gene expression in mice testes exposed to $1.765 \mathrm{GHz}$ microwave in utero. J Rep Med 62: 324-328. PMID: 30028096 
Li HW, Xie Y, Li F, Sun GC, Chen Z, Zeng HS (2016) Effect of miR-19a and miR-21 on the JAK/STAT signaling pathway in the peripheral blood mononuclear cells of patients with systemic juvenile idiopathic arthritis. Exp Ther Med 11: 2531-2536. https://doi. org/10.3892/etm.2016.3188

Li X, Yang J, Wang X, Li X, Liang J, Xing H (2016) Role of TWIST2, E-cadherin and Vimentin in epithelial ovarian carcinogenesis and prognosis and their interaction in cancer progression. Eur J Gynaecol Oncol 37: 100-108. PMID: 27048119

Li Y, Qin C (2019) MiR-1179 inhibits the proliferation of gastric cancer cells by targeting HMGB1. Hum Cell 1-8.

Lin C, Hu Z, Yuan G, Su H, Zeng Y, Guo Z, Zhong F, Jiang K, He S (2018) MicroRNA-1179 inhibits the proliferation, migration and invasion of human pancreatic cancer cells by targeting E2F5. Chem Biol Int 291: 65-71. https://doi.org/10.1016/j.cbi.2018.05.017

Liu L, Yu X, Guo X, Tian Z, Su M, Long Y, Huang C, Zhou F, Liu $\mathrm{M}$, Wu X, Wang X (2012) miR-143 is downregulated in cervical cancer and promotes apoptosis and inhibits tumor formation by targeting Bcl-2. Mol Med Rep 5: 753-760. https://doi.org/10.3892/ mmr.2011.696

Liu T, Wei J, Jiang C, Wang C, Zhang X, Du Y, Li J, Zhao H (2017) $\mathrm{CHAF} 1 \mathrm{~A}$, the largest subunit of the chromatin assembly factor 1 complex, regulates the growth of H1299 human non-small cell lung cancer cells by inducing G0/G1 cell cycle arrest. Exp Ther Med 14: 4681-4686. https://doi.org/10.3892/etm.2017.5201

Luo Y, Wang Q, Tian P, Jia Y (2017) Highly expressed CHAF1A and PCNA are positively associated with malignancy of cervical squamous cell carcinoma. Chin J Cell Mol Immunol 33: 1696-1701 (in Chinense Xi Bao Yu Fen Zi Mian Yi Xue Za Zhi)

Markham MJ, Wachter K, Agarwal N, Bertagnolli MM, Chang SM, Dale W, Diefenbach CSM, Rodriguez-Galindo C, George DJ, Gilligan TD, Harvey RD, Johnson ML, Kimple RJ, Knoll MA, LoConte N, Maki RG, Meisel JL, Meyerhardt JA, Pennell NA, Rocque GB, Sabel MS, Schilsky RL, Schneider BJ, Tap WD, Uzzo RG, Westin SN (2020) Clinical Cancer Advances 2020: Annual Report on Progress Against Cancer From the American Society of Clinical Oncology. J Clin Oncol 38: 1081. https://doi.org/10.1200/jco.19.03141

Peña C, García JM, García V, Silva J, Domínguez G, Rodríguez R, Maximiano C, García de Herreros A, Muñoz A, Bonilla F (2006) The expression levels of the transcriptional regulators p300 and CtBP modulate the correlations between SNAIL, ZEB1, E-cadherin and vitamin $\mathrm{D}$ receptor in human colon carcinomas. Int J Cancer 119: 2098-2104. https://doi.org/10.1002/ijc.22083

Peng H, B Du, H Jiang,J Gao (2016) Over-expression of CHAF1A promotes cell proliferation and apoptosis resistance in glioblastoma cells via AKT/FOXO3a/Bim pathway. Biochem Biophys Res Commun 469: 1111-1116. https://doi.org/10.1016/j.bbrc.2015.12.111

Qu X, Zhu L, Song L, Liu S (2020) circ_0084927 promotes cervical carcinogenesis by sponging miR-1179 that suppresses CDK2, a cell cycle-related gene. Cancer Cell Int. 20: 333. https://doi.org/10.1186/ s12935-020-01417-2

Sánchez-Tilló E, de Barrios O, Siles L, Cuatrecasas M, Castells A, Postigo A (2011) $\beta$-catenin/TCF4 complex induces the epithelialto-mesenchymal transition (EMT)-activator ZEB1 to regulate tumor invasiveness. Proc Natl Acad Sci U S A 108: 19204-19209. https:// doi.org/10.1073/pnas.1108977108

Song B, Du J, Song DF, Ren JC, Feng Y (2018) Dysregulation of NCAPG, KNL1, miR-148a-3p, miR-193b-3p, and miR-1179 may contribute to the progression of gastric cancer. Biol Res 51: 44. https://doi.org/10.1186/s40659-018-0192-5
Song L, Dai Z, Zhang S, Zhang H, Liu C, Ma X, Liu D, Zan Y, Yin $\mathrm{X}$ (2018) MicroRNA-1179 suppresses cell growth and invasion by targeting sperm-associated antigen 5-mediated Akt signaling in human non-small cell lung cancer. Biochem Biophys Res Commun 504: 164-170. https://doi.org/10.1016/j.bbrc.2018.08.149

Sun S, Yang X, Qin X, Zhao Y (2020) TCF4 promotes colorectal cancer drug resistance and stemness via regulating ZEB1/ZEB2 expression. Protoplasma 257: 921-930. https:/ /doi.org/10.1007/s00709020-01480-6

Wang F, Li Y, Zhou J, Xu J, Peng C, Ye F, Shen Y, Lu W, Wan $\mathrm{X}$, Xie X (2011) miR-375 is down-regulated in squamous cervical cancer and inhibits cell migration and invasion via targeting transcription factor SP1. Am J Pathol 179: 2580-2588. https://doi. org/10.1016/j.ajpath.2011.07.037

Wilting SM, van Boerdonk RA, Henken FE, Meijer CJ, Diosdado B, Meijer GA, le Sage C, Agami R, Snijders PJ, Steenbergen RD (2010) Methylation-mediated silencing and tumour suppressive function of hsa-miR-124 in cervical cancer. Mol Cancer 9: 167. https:// doi.org/10.1186/1476-4598-9-167

Wu Z, Cui F, Yu F, Peng X, Jiang T, Chen D, Lu S, Tang H, Peng Z (2014) Up-regulation of CHAF1A, a poor prognostic factor, facilitates cell proliferation of colon cancer. Biochem Biophys Res Commun 449: 208-215. https://doi.org/10.1016/j.bbrc.2014.05.006

Xia D, Yang X, Liu W, Shen F, Pan J, Lin Y, Du N, Sun Y, Xi X (2017) Over-expression of CHAF1A in Epithelial Ovarian Cancer can promote cell proliferation and inhibit cell apoptosis. Biochem Biophys Res Commun 486: 191-197. https://doi.org/10.1016/j. bbrc.2017.03.026

Xiao H, Yu L, Li F, Wang H, Li W, He X (2018) MiR-340 suppresses the metastasis by targeting EphA3 in cervical cancer. Cell Biol Int 42: 1115-1123. https://doi.org/10.1002/cbin.10974

Xie H, Zhao Y, Caramuta S, Larsson C, Lui WO (2012) miR-205 expression promotes cell proliferation and migration of human cervical cancer cells. PLoS One. 7: e46990. https://doi.org/10.1371/ journal.pone.0046990

Ye M, Hou H, Shen M, Dong S, Zhang T (2020) Circular RNA circFOXM1 plays a role in papillary thyroid carcinoma by sponging miR-1179 and regulating HMGB1 expression. Mol Ther Nucleic Acids 19: 741-750. https://doi.org/10.1016/j.omtn.2019.12.014

Yu P, Shen X, Yang W, Zhang Y, Liu C, Huang T (2018) ZEB1 stimulates breast cancer growth by up-regulating hTERT expression. Biochem Biophys Res Commun 495: 2505-2511. https://doi. org/10.1016/j.bbrc.2017.12.139

Zhang P, Sun Y, Ma L (2015) ZEB1: at the crossroads of epithelialmesenchymal transition, metastasis and therapy resistance. Cell Cycle 14: 481-487. https://doi.org/10.1080/15384101.2015.1006048

Zheng L, Liang X, Li S, Li T, Shang W, Ma L, Jia X, Shao W, Sun P, Chen C, Jia J (2018) CHAF1A interacts with TCF4 to promote gastric carcinogenesis via upregulation of c-MYC and CCND1 expression. EBioMedicine 38: 69-78. https://doi.org/10.1016/j.ebiom.2018.11.009

Zhihong Z, Rubin C, Liping L, Anpeng M, Hui G, Yanting W, Zhenxiu S (2019) MicroRNA-1179 regulates proliferation and chemosensitivity of human ovarian cancer cells by targeting the PTENmediated PI3K/AKT signaling pathway. Arch Med Sci 16: 907-914. https://doi.org/10.5114/aoms.2019.86798

Zhu X, Er K, Mao C, Yan Q, Xu H, Zhang Y, Zhu J, Cui F, Zhao W, Shi H (2013) miR-203 suppresses tumor growth and angiogenesis by targeting VEGFA in cervical cancer. Cell Pbysiol Biochem 32: 64-73. https://doi.org/10.1159/000350125 